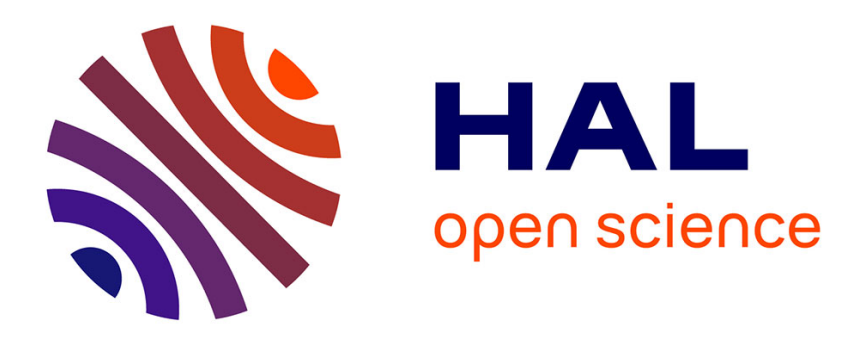

\title{
A New W Ursae Majoris-type Binary Star System in the Constellation Phoenix
}

\author{
Salvador Barquin
}

\section{To cite this version:}

Salvador Barquin. A New W Ursae Majoris-type Binary Star System in the Constellation Phoenix. Research Notes of the AAS, 2019, 3 (3), pp.53. 10.3847/2515-5172/ab108e . hal-02089503

\section{HAL Id: hal-02089503 https://hal.science/hal-02089503}

Submitted on 13 Apr 2019

HAL is a multi-disciplinary open access archive for the deposit and dissemination of scientific research documents, whether they are published or not. The documents may come from teaching and research institutions in France or abroad, or from public or private research centers.
L'archive ouverte pluridisciplinaire HAL, est destinée au dépôt et à la diffusion de documents scientifiques de niveau recherche, publiés ou non, émanant des établissements d'enseignement et de recherche français ou étrangers, des laboratoires publics ou privés. 
Published in RNAAS, 3, 53

doi:10.3847/2515-5172/ab108e

\title{
A New W Ursae Majoris-type Binary Star System in the Constellation Phoenix
}

\author{
Salvador Barquin ${ }^{1}$ \\ 1) E-39700 Castro-Urdiales, Cantabria, Spain \\ salvadorbarquin@outlook.com
}

Keywords: binaries: close — methods: data analysis — stars: individual (UCAC4 234-184364) — techniques: photometric

A new W Ursae Majoris-type binary star system was discovered at R.A. $=23^{\mathrm{h}} 59^{\mathrm{m}} 14.502^{\mathrm{s}}$, Decl. $=-43^{\circ} 21^{\prime} 59.258^{\prime \prime}$. It was detected during a search through the All Sky Automated Survey for SuperNovae (ASAS-SN, Shappee et al., 2014; Kochanek et al., 2017) database for previously unreported eclipsing binary stars in the upper right area of the constellation Phoenix, chosen for this search due to its abundance of non-crowded fields.

First of all, an area with a radius of 13 arcminutes was randomly selected, with the star cataloged as GSC 07529-00536 in the Guide Star Catalog (Morrison et al., 2001) in its center. Then, in order to get the list of stars located in this area, the Fourth U.S. Naval Observatory CCD Astrograph Catalog (Zacharias et al., 2012) was consulted through the VizieR database of astronomical catalogs (Ochsenbein et al., 2000). Thereafter, only those stars brighter than 15 mag in the $V$-band were elected to check their variability, retrieving their light curves from the ASAS-SN database by querying through its website, ${ }^{1}$ with the intention of detecting a new binary star system.

The analysis of photometry data was performed using the ANOVA algorithm (Schwarzenberg-Czerny, 1996) implemented in the software PERANSO 2.51 (Paunzen \& Vanmunster, 2016) for the search of possible periodicities. As a result of this, only one star cataloged as UCAC4 234-184364 (R.A. $=23^{\mathrm{h}} 59^{\mathrm{m}} 14.502^{\mathrm{s}}$, decl. $=-43^{\circ} 21^{\prime} 59.258^{\prime \prime}$ ) was clearly identified as a variable star regarding its lightcurve. The retrieved data for this star, obtained by the quadruple $14 \mathrm{~cm}$ "Cassius" telescope located in Cerro Tololo, Chile, and the quadruple $14 \mathrm{~cm}$ "Payne-Gaposchkin" telescope located in Sutherland, South Africa, covered 872 data points from HJD 2,456,777.90505 to $2,458,380.76177$ with a mean photometric error of 0.049 mag in the $V$-band.

After that, the position of the variable star was checked in the International Variable Star Index (Watson et al., 2006) and in the General Catalog of Variable Stars (Samus' et al., 2017), where it was still unreported in both. Following this, and having already previously estimated the period $P=0.265301 \pm 0.000001$ days, the software VSTAR 2.19.0 (Benn, 2012) was then used to determine the primary minimum epoch HJD 2,456,995.57424 \pm 0.0001 (through its polynomial fitting algorithm tool), proceeding to create the phase curve (Figure 1) that shows how the $V$ Magnitude varies during the orbital cycle.

\footnotetext{
${ }^{1}$ https://asas-sn.osu.edu/
} 


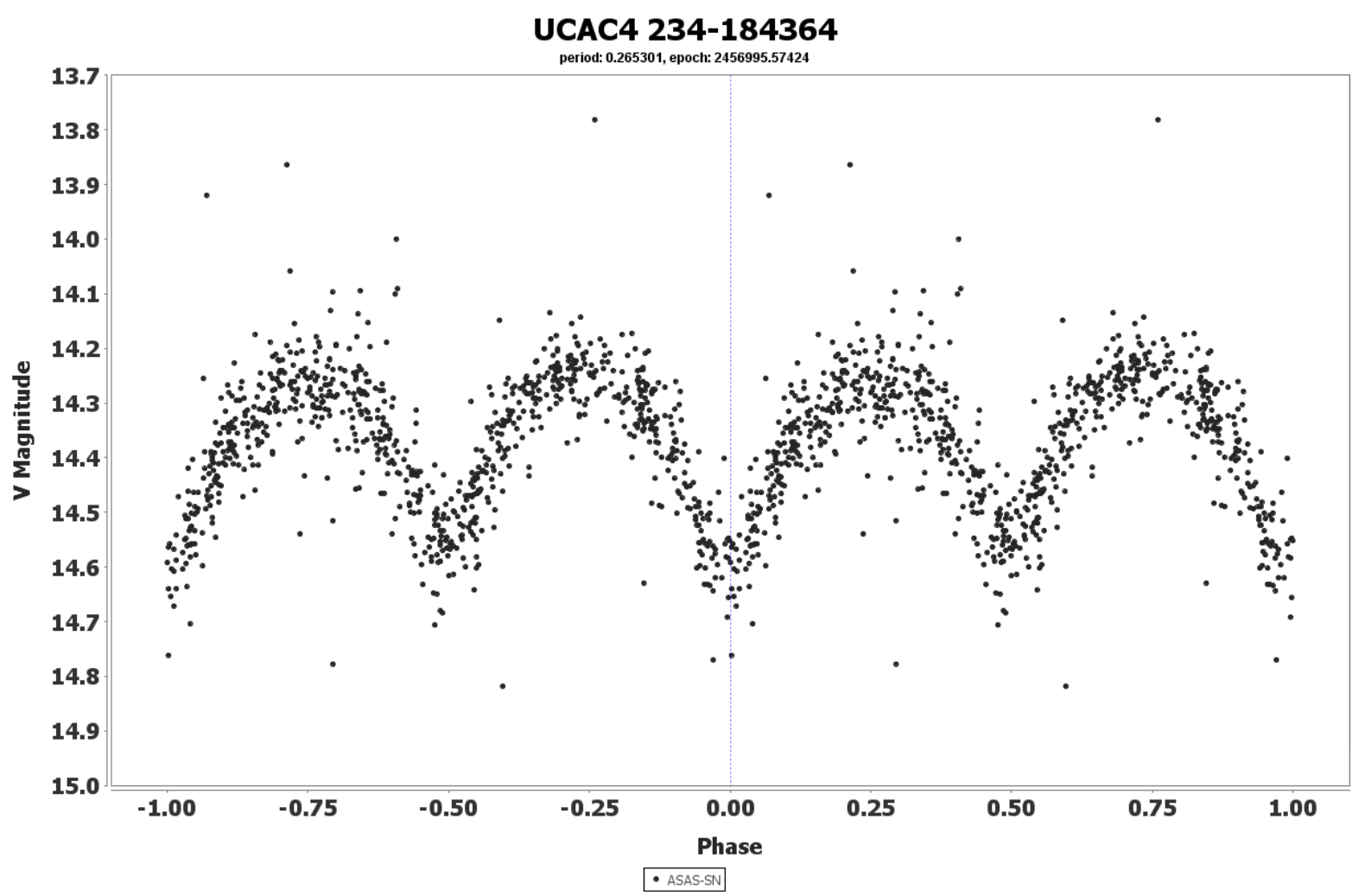

Figure 1. Phase curve of UCAC4 234-184364 constructed with V-band observations from the ASAS-SN database for period $P=0.265301$ days and epoch HJD 2,456,995.57424

The magnitude measurement of the star ranges from 14.23 mag in the $V$-band at its brightest to $14.62 \mathrm{mag}$ in the $V$-band at its faintest. The shape of the phase curve and its characteristics implies that this new variable star is an eclipsing binary of W Ursae Majoris type, an overcontact binary star system with a common envelope for its two components.

Among the cross-identifications corresponding to this star are GSC 08021-00559 from the Guide Star Catalog and USNO-B1.0 0466-0781404 from the USNO-B Catalog (Monet et al., 2003).

This research has made use of the VizieR database operated by the Centre de Données Astronomiques in Strasbourg, France.

\section{References}

Benn, D. 2012, JAVSO, 40, 852, 2012JAVSO..40..852B

Kochanek, C. S. et al., 2017, PASP, 129, 104502, 2017PASP..129j4502K

Monet, D. G. et al., 2003, AJ, 129, 984, 2003AJ....125..984M

Morrison, J. E. et al., 2001, AJ, 121, 1752, 2001AJ....121.1752M

Ochsenbein, F. et al., 2000, A\&AS, 143, 23, 2000A\&AS..143...230

Paunzen, E., Vanmunster, T., 2016, 2016, AN, 337, 239, 2016AN...337..239P

Samus', N. N. et al., 2017, ARep, 61, 80, 2017ARep...61...80S

Schwarzenberg-Czerny, A., 1996, ApJL, 460, L107, 1996ApJ...460L.107S

Shappee, B. J. et al., 2014, ApJ, 788, 48, 2014ApJ...788...48S

Watson, C. L. et al., 2006, Society for Astronomical Sciences Annual Symposium, 25, 47, 2006SASS...25...47W

Zacharias, N. et al., 2012, VizieR On-line Data Catalog: I/322A, 2012yCat.1322....0Z 